\title{
Typal Heterogeneous Equality Types
}

\author{
ANDREW M. PITTS, University of Cambridge, UK
}

The usual homogeneous form of equality type in Martin-Löf Type Theory contains identifications between elements of the same type. By contrast, the heterogeneous form of equality contains identifications between elements of possibly different types. This short note introduces a simple set of axioms for such types. The axioms are shown to be equivalent to the combination of systematic elimination rules for both forms of equality, albeit with typal (also known as "propositional") computation properties, together with Streicher's Axiom K, or equivalently, the principle of uniqueness of identity proofs.

CCS Concepts: • Theory of computation $\rightarrow$ Type theory;

Additional Key Words and Phrases: Type theory, equality types, heterogeneous equality

\section{ACM Reference format:}

Andrew M. Pitts. 2020. Typal Heterogeneous Equality Types. ACM Trans. Comput. Logic 21, 3, Article 25 (April 2020), 10 pages.

https://doi.org/10.1145/3379447

\section{INTRODUCTION}

Equality types in the intensional version of Martin-Löf Type Theory (see, for example, Reference [12, Section 8.1]) are traditionally formulated in terms of an introduction rule (reflexivity) together with a rule for eliminating proofs of equality and a rule describing how elimination computes when it meets a reflexivity proof. Some recent work [4, 7] on models of Homotopy Type Theory [17] uses a formulation of equality types that differs from this in two respects. First, the elimination operation is replaced by the combination of a simple operation for transporting elements along proofs of equality, together with an axiom asserting contractibility of singleton types. Second, the analogue of the computation rule for the eliminator, namely, that transporting along a reflexivity proof does nothing, is weakened from a judgemental equality to the existence of an element of the corresponding equality type; see Reference [9] and Figure 2 in Reference [4]. This formulation is sometimes called a "propositional" equality type [18], but here I will follow Shulman [14, Section 1.6] for the reasons given there and refer to typal equality types. Although these changes to the formulation of equality types affect computation, it seems that they do not change what is provable (see Reference [5], for example) and they make it easier to construct models. Furthermore, they can lead to surprising simplifications. For example, Lumsdaine [private communication] has observed that the computation rule is superfluous (for elimination, but the observation also holds for transport): If a proto-identity type has a transport operation lacking its

Authors' address: A. M. Pitts, University of Cambridge, Dept. Computer Science \& Technology, Cambridge, CB3 0FD, UK; email: andrew.pitts@cl.cam.ac.uk.

Permission to make digital or hard copies of all or part of this work for personal or classroom use is granted without fee provided that copies are not made or distributed for profit or commercial advantage and that copies bear this notice and the full citation on the first page. Copyrights for components of this work owned by others than the author(s) must be honored. Abstracting with credit is permitted. To copy otherwise, or republish, to post on servers or to redistribute to lists, requires prior specific permission and/or a fee. Request permissions from permissions@acm.org.

(C) 2020 Copyright held by the owner/author(s). Publication rights licensed to ACM.

1529-3785/2020/04-ART25 \$15.00

https://doi.org/10.1145/3379447 


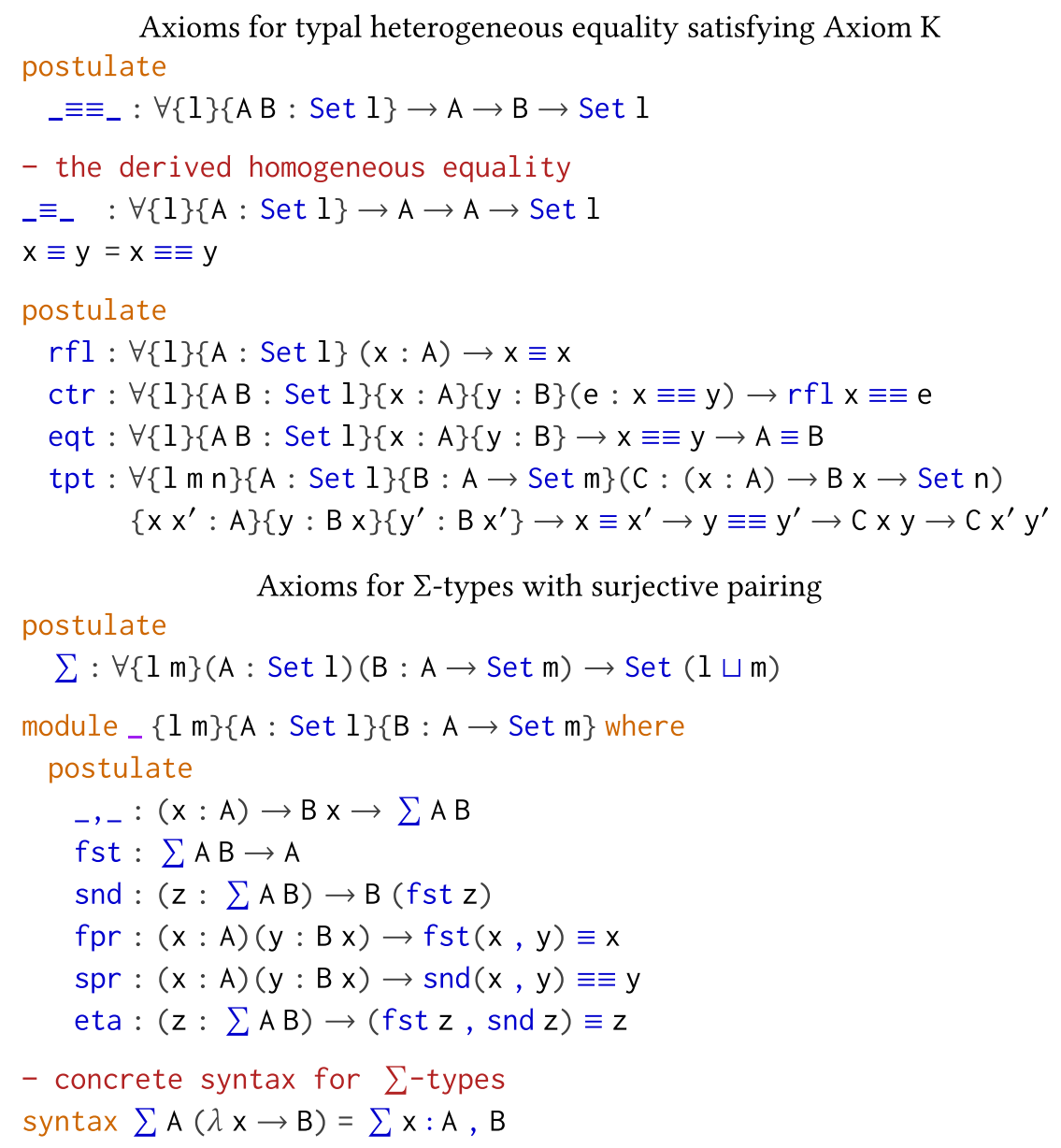

Fig. 1. The Axioms.

typal computation property, then the operation can be corrected to a new one that does have the computation property (see Lemma 2.1 and the Appendix).

The above remarks apply to the usual, homogeneous notion of equality in which elements of the same type are compared. The purpose of this article is to give an analogous treatment of heterogeneous equality $[2,11]$ in the presence of $\Sigma$-types and the Axiom K of Streicher [15, Section 1.2]. Since Axiom K is not compatible with the Univalence Principle of Homotopy Type Theory [17, Example 3.1.9], the focus here is on the simpler (but still useful!) world of zero-dimensional type theory. We will see that the axioms in Figure 1 capture homogeneous and heterogeneous equality satisfying their usual dependent elimination and (typal) computation properties and Axiom K, and $\Sigma$-types with their usual dependent elimination and (typal) computation properties. It seems necessary to include $\Sigma$-types to get Lumsdaine's result mentioned above (see Remark 2.5); the axioms we give for such types are standard, except that the equality property of dependent second projection (spr) is simplified by the use of heterogeneous rather than homogeneous equality. The axioms in the figure are pleasingly simple compared to the usual formulation in terms of elimination and computation properties, and may aid finding new models of heterogeneous equality types. 
The implementation of intensional Martin-Löf Type Theory provided by Agda 2.6 [1] is used to state the axioms and develop their properties. More precisely, we just make use of Agda's implementation of a countably infinite, non-cumulative hierarchy of universes Set 1, where 1 ranges over a type Level of universe levels whose closed normal forms are in bijection with the natural numbers. The universes are closed under dependent function types (written in Agda as $(x: A) \rightarrow B$ ) and inductive types. The use of a whole hierarchy of universes is necessary; for example, the function eqt in Figure 1 takes a heterogeneous equality type $x \equiv \equiv y$ in universe Set $l$ and produces a homogeneous one $A \equiv B$ in the universe one level up, which is denoted Set(lsuc l) in Agda. We also use Agda's notation for infix and for implicit arguments. For example, the function _E三 in Figure 1 takes five arguments, the first three of which are implicit and the last two of which are infix. In particular, Agda's ability to infer the values of implicit arguments (or of unspecified explicit arguments, which are denoted by an underscore, ${ }_{-}$) is used quite aggressively in what follows, to be able to see the wood from the trees.

Although the code in this article has been checked by Agda, some parts of it that are not essential for understanding the development have been hidden; the complete (non-literate) Agda code can be found at https://doi.org/10.17863/CAM.47902.

\section{THE AXIOMS AND THEIR PROPERTIES}

Figure 1 postulates a family of types _ $\equiv \equiv{ }_{-}$in all universes, together with some operations on them that together capture a typal version of heterogeneous equality. Heterogeneous equality types were introduced by McBride [11, Section 5.1.3] under the name of "John Major equality." Unlike ordinary, homogeneous equality types, such a type $\mathrm{x} \equiv \equiv \mathrm{y}$ relates elements $\mathrm{x}$ and $\mathrm{y}$ of possibly different types, A and B say. The intention is that elements of type $x \equiv \equiv y$ denote proofs that not only are $x$ and $y$ equal, but so also are their types $A_{\text {and }}$ B. The figure defines homogeneous equality _ $\equiv_{-}$as the special case of _E三_ when the types of the two arguments are already known to be the same. Axiom $\mathrm{rfl}$ says that $\equiv$ is reflexive. Axiom $\mathrm{ctr}$ is a heterogeneous version of the contractibility property of singleton types (cf. center in Figure 2 of [4]). Axiom eqt says that heterogeneously equal things have (homogeneously) equal types. Axiom tpt is a form of the transport property of equality (cf. T in Figure 2 of Reference [4]) involving both homogeneous and heterogeneous equalities. Finally, $\sum_{\text {, }_{-},-}, \mathrm{fst}$, snd, fpr, spr and eta axiomatize dependent product types satisfying surjective pairing.

We begin with some simple lemmas establishing the basics of equational logic for $\equiv \equiv$, namely, chain-reasoning using reflexivity (already an axiom), symmetry, transitivity, and congruence properties. These are given in Figure 2.

The axioms in Figure 1 are notably lacking a "regularity" property for tpt, that is, a proof of type tpt $(r f l x)(r f l y) z \equiv z$. But such a thing is needed if we are to derive the expected elimination and (typal) computation rules for $\_\equiv \equiv_{-}$and _ $\equiv_{-}$. To get those, one can define a "corrected" form of transport that has this regularity property, using a simplified version of a trick due to Peter Lumsdaine [unpublished]. In fact, it is enough to produce a function coercing proofs of equality of types $\mathrm{e}: \mathrm{A} \equiv \mathrm{B}$ into functions coe $\mathrm{e}: \mathrm{A} \rightarrow \mathrm{B}$ and which satisfies the heterogeneous regularity property that coe $\mathrm{x} \equiv \equiv \times$ (so that, given how we define $\equiv$ in terms of $\equiv \equiv$, the usual form of regularity, coe $(r f l A) x \equiv x$, is just the special case of this when e is $r f l A)$.

LEMma 2.1. The axioms in Figure 1 imply the existence of a coercion function

coe : $\forall\{1\}\{A B:$ Set 1$\} \rightarrow A \equiv B \rightarrow A \rightarrow B$

satisfying a heterogeneous regularity property:

coeIsRegular : $\forall\{1\}\{A B:$ Set 1$\}(e: A \equiv B)(x: A) \rightarrow$ coe e $x \equiv \equiv x$ 


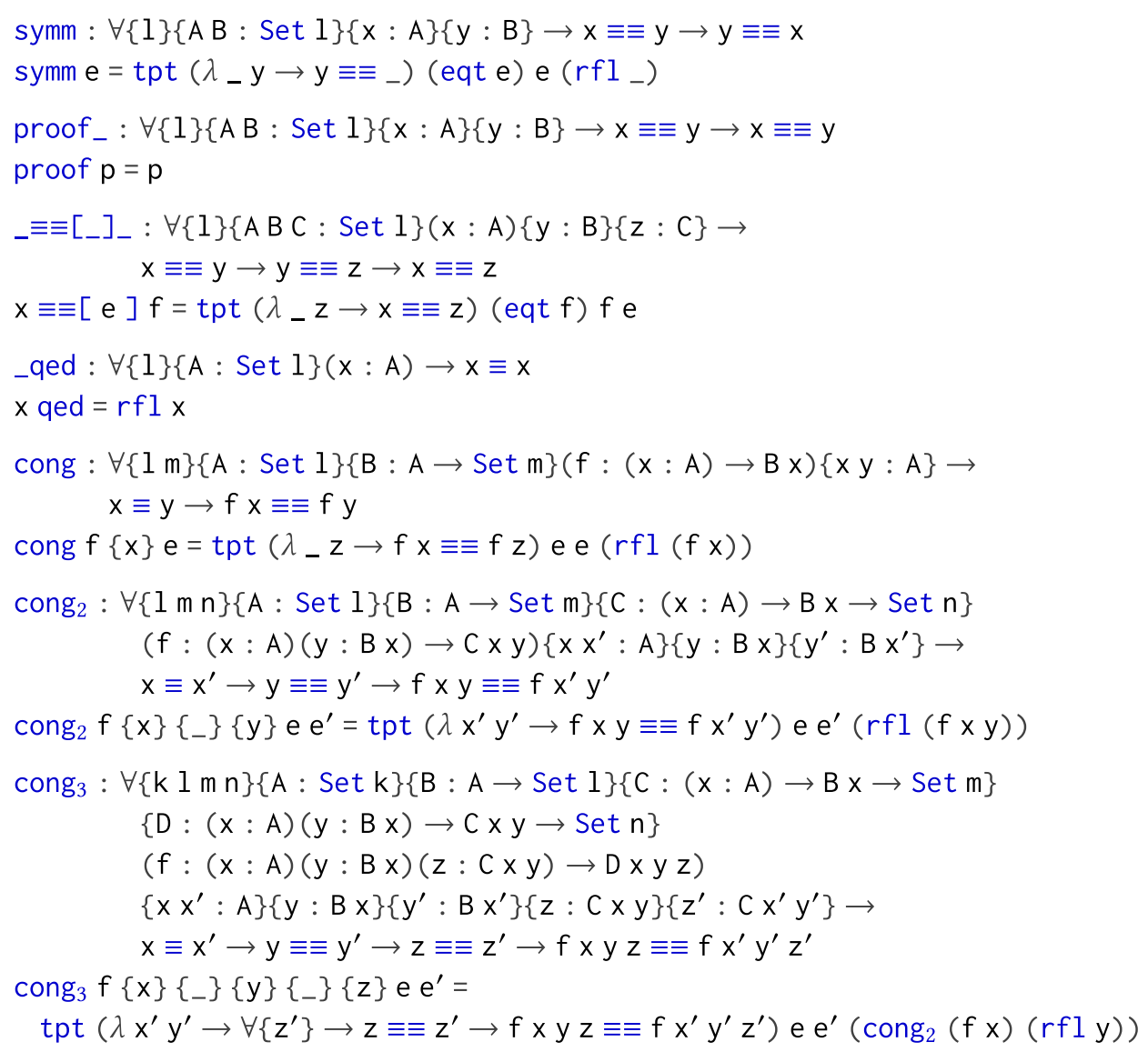

Fig. 2. Equational reasoning for heterogeneous equality.

Proof. First, we define the type of functions that are injective with respect to $\equiv$ and note that the identity function is one such:

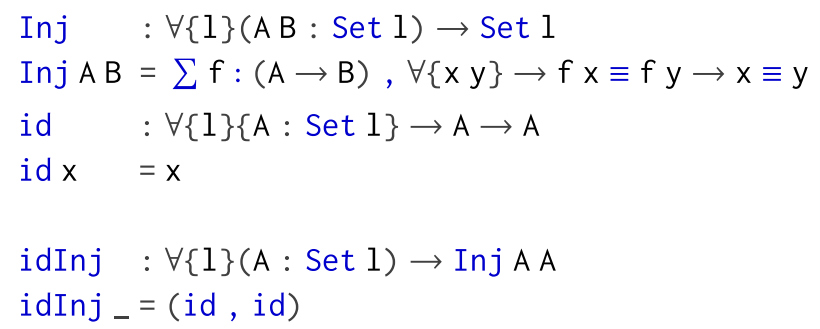

Next, we use tpt to define a function coercing equalities into injective functions:

icoe : $\forall\{1\}\{A B:$ Set 1$\} \rightarrow A \equiv B \rightarrow \operatorname{Inj} A B$

icoe $\{1\}\{A\}$ e $=\operatorname{tpt}\left(\lambda_{-} C \rightarrow \operatorname{Inj} A C\right)(\operatorname{rfl}($ Set 1$))$ e $(\operatorname{idInj} A)$

The injectiveness of icoe e is used as follows. Applying the operation tpt to the type family fsticoe: $\forall\{1\}\{A:$ Set 1$\}(x: A)(B:$ Set 1$)(e: A \equiv B) \rightarrow$ Set 1

fsticoe $x B e=\sum y: B,(f s t($ icoe $(r f l B)) y \equiv f s t($ icoe e $) x)$ 
we can transport the element $(e, r f l(f s t(i c o e(r f l A)) x)$ of type fsticoe $x A(r f l A)$ along e : $A \equiv B$ and ctr e $: r f l A \equiv \equiv$ e to give an element of type fsticoe $\times B$ e. The first projection of this element gives the value of the desired coercion along e at $x$ :

coe e $x=f s t\left(\right.$ tpt $($ fsticoe $x)$ e $\left.(\operatorname{ctr} e)\left(x, r f l_{-}\right)\right)$

and its second projection can be used along with the injectiveness property of icoe to get the regularity property of this coercion:

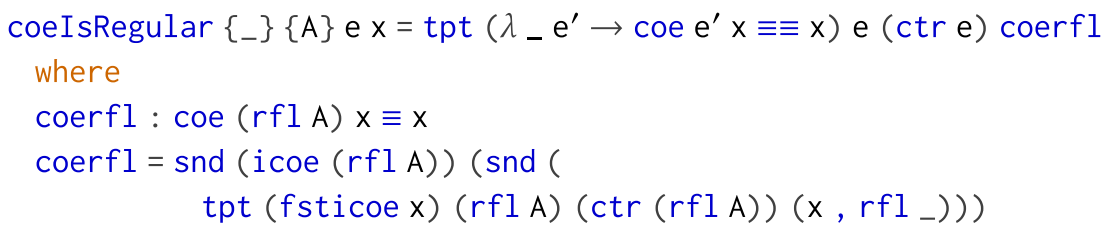

An immediate corollary is that the axioms imply the uniqueness of identity proofs (UIP) and hence Streicher's Axiom K [15]. (We will see in Section 3 that in fact it is only the tpt function that contains an implicit use of Axiom K.)

Theorem 2.2 (UIP AND Axiom K). The axioms in Figure 1 imply that $\equiv$ satisfies

$$
: \forall\{1\}\{A: \text { Set } 1\}\{x y: A\}\left(e e^{\prime}: x \equiv y\right) \rightarrow e \equiv e^{\prime}
$$

axiomk

$$
\begin{aligned}
& : \forall\{1 \mathrm{~m}\}\{A: \text { Set } 1\}\{x: A\}(P: x \equiv x \rightarrow \operatorname{Set} m)(p: P(r f l x)) \rightarrow \\
& \quad \forall e \rightarrow P e
\end{aligned}
$$

axiomKComp : $\forall\{1 \mathrm{~m}\}\{\mathrm{A}:$ Set 1$\}\{\mathrm{x}: \mathrm{A}\}(\mathrm{P}: \mathrm{x} \equiv \mathrm{x} \rightarrow \operatorname{Set} \mathrm{m})(\mathrm{p}: \mathrm{P}(\mathrm{rfl} \mathrm{x})) \rightarrow$ axiomk P $p(r f l x) \equiv p$

Proof. Using the functions from Figure 2 and Lemma 2.1, we have

uipe e $\quad=\operatorname{tpt}\left(\lambda_{-} \mathrm{e}^{\prime \prime} \rightarrow \mathrm{e}^{\prime \prime} \equiv \equiv \mathrm{e}^{\prime}\right)$ e $(\operatorname{ctr} \mathrm{e})\left(\operatorname{ctr} \mathrm{e}^{\prime}\right)$

axiomk P pe $=\operatorname{coe}\left(\operatorname{cong}_{2}\left(\lambda_{-} \rightarrow \mathrm{P}\right)(\mathrm{rfl} \mathrm{p})(\operatorname{ctr} \mathrm{e})\right) \mathrm{p}$

axiomkComp P p $=$ coeIsRegular $\left(\operatorname{cong}_{2}\left(\lambda_{-} \rightarrow \mathrm{P}\right)(\mathrm{rfl} p)\left(\operatorname{ctr}\left(\mathrm{rfl}_{-}\right)\right)\right) \mathrm{p}$

The elimination and computation properties of $\equiv$ and $\equiv \equiv$ then follow:

Theorem 2.3 (Elimination AND typal Computation Properties). The axioms in Figure 1 imply that $\equiv$ has the usual elimination and (typal) computation properties of homogeneous equality (in the form suggested by Reference [13])

$$
\begin{aligned}
& \equiv E \lim : \forall\{1 \mathrm{~m}\}\{\mathrm{A}: \text { Set } 1\}\{\mathrm{x}: A\}(P:(y: A) \rightarrow \mathrm{x} \equiv \mathrm{y} \rightarrow \text { Set } \mathrm{m}) \\
& (p: P x(r f l x))(y: A)(e: x \equiv y) \rightarrow P \text { y e } \\
& \equiv \text { Comp : } \forall\{1 \mathrm{~m}\}\{A: \text { Set } 1\}\{x: A\}(P:(y: A) \rightarrow x \equiv y \rightarrow \text { Set } m) \\
& (\mathrm{p}: \mathrm{P} \times(\mathrm{rfl} \mathrm{x})) \rightarrow \equiv E \operatorname{Elim} \mathrm{P} \mathrm{p} \times(\mathrm{rfl} \mathrm{x}) \equiv \mathrm{p}
\end{aligned}
$$

The axioms also imply that $\equiv \equiv$ has the elimination and (typal) computation properties of heterogeneous equality described by McBride [11, Section 5.1.3]

$$
\begin{aligned}
& \equiv \equiv \operatorname{Elim}: \forall\{1 \mathrm{~m}\}\{A: \text { Set } 1\}\{x: A\}(P:(B: \text { Set } 1)(y: B) \rightarrow x \equiv \equiv y \rightarrow \text { Set } m) \\
& (p: P A x(r f l x))(B: \operatorname{Set} 1)(y: B)(e: x \equiv \equiv y) \rightarrow P B \text { y e } \\
& \equiv \equiv \text { Comp : } \forall\{1 \mathrm{~m}\}\{\mathrm{A}: \text { Set } 1\}\{\mathrm{x}: \mathrm{A}\}(\mathrm{P}:(B: \operatorname{Set} 1)(\mathrm{y}: \mathrm{B}) \rightarrow \mathrm{x} \equiv \equiv \mathrm{y} \rightarrow \operatorname{Set} \mathrm{m}) \\
& (p: P A \times(r f l x)) \rightarrow \equiv \equiv E \lim P p A x(r f l x) \equiv p
\end{aligned}
$$


Proof. Using the functions from Figure 2 and Lemma 2.1, we have

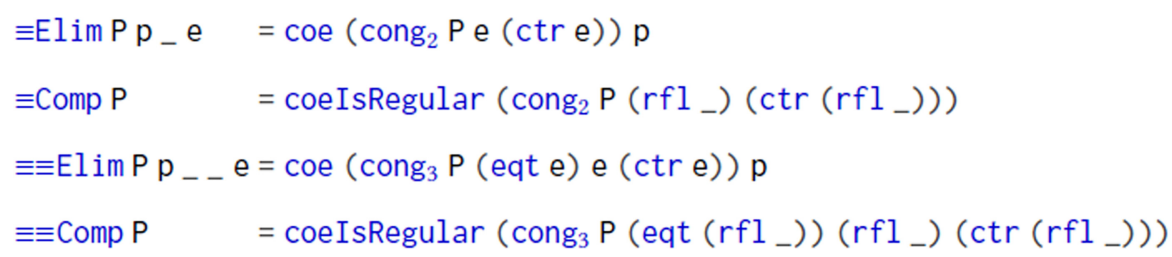

Note that a corollary of the above two theorems is that ${ }_{-} \equiv_{-}$is uniquely determined up to logical equivalence by the axioms in Figure 1. In other words, for any other such family of types ${ }_{\text {_ }} \equiv \equiv^{\prime}{ }_{\text {_ }}$, there are functions in either direction between $\mathrm{x} \equiv \equiv \mathrm{y}$ and $\mathrm{x} \equiv \equiv^{\prime} \mathrm{y}$; and because of UIP, these are necessarily mutually inverse up to $\equiv \equiv\left(\right.$ or $\left.\equiv \equiv^{\prime}\right)$.

Remark 2.4. $\equiv \equiv E$ Elim is the elimination form systematically derived [3] from _ $\equiv \equiv{ }_{-}$and $r f$, regarding them as the formation and introduction rules for an inductive type. As McBride [11, page 120] points out, $\equiv \equiv E$ I im is not very useful because of the way its motive $P$ involves abstraction over an arbitrary type B. McBride goes on to give another, more useful form of elimination for $\equiv \equiv$, but in our setting where $\equiv$ is a special case of $\equiv \equiv$, that coincides with the eliminator $\equiv$ Elim.

Remark 2.5 (The role of $\Sigma$-types). One of the strengths of machine-checked mathematics is that it aids the detection of logical dependency. Although we included the equations fpr, spr, and eta for $\Sigma$-types in Figure 1, they have not been used for the results so far, as may be verified by commenting them out from this literate Agda file and re-checking it up to this point.

So only the weak form of dependent product given by $\sum,,_{-}$, , fst and snd in the figure is used to define the regular version of coercion in Lemma 2.1 and then prove Theorems 2.2 and 2.3. It would be nice if there was some way to define $\sum,_{-},{ }_{-}, f s t$, and snd just using dependent function types and universes.

However, the extra equations fpr, spr, and eta for $\sum$ are of course very natural. Let us record the fact that they enable one to define the usual elimination rule for dependent products, with a typal computation rule:

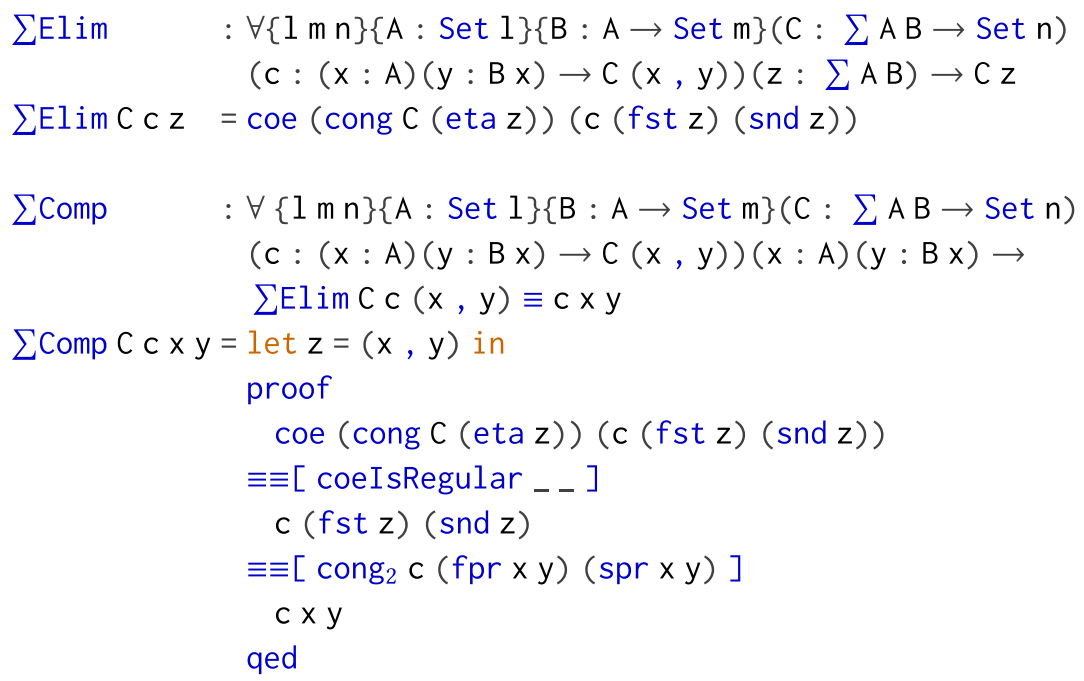




\section{CONSISTENCY OF THE AXIOMS}

We have seen that the axioms in Figure 1 suffice to define dependent products and both heterogeneous and homogeneous equality types with uniqueness of identity proofs, all satisfying the usual elimination properties, albeit with typal computation rules. Conversely, it is not hard to see that the elimination and computation rules in Theorem 2.3 and Remark 2.5, together with Axiom K, imply the axioms in Figure 1. Instead of doing that, in this section, we just check that the axioms are provable from inductive definitions of equality and dependent product types. One can make these inductive definitions in Agda as follows:

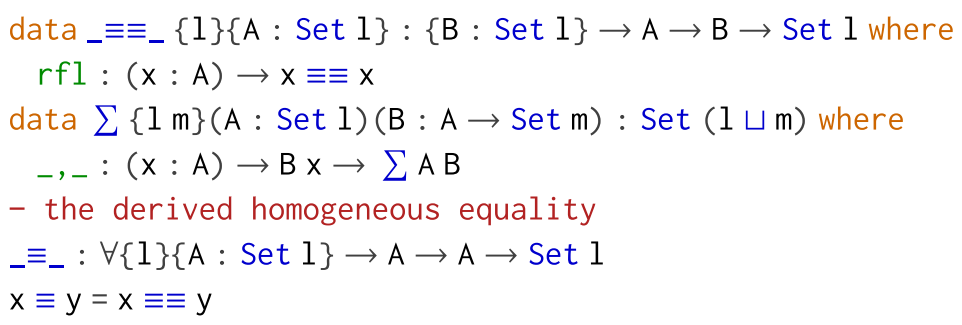

Then Agda's implementation of dependent pattern matching enables straightforward definitions of the functions from Figure 1, as follows:

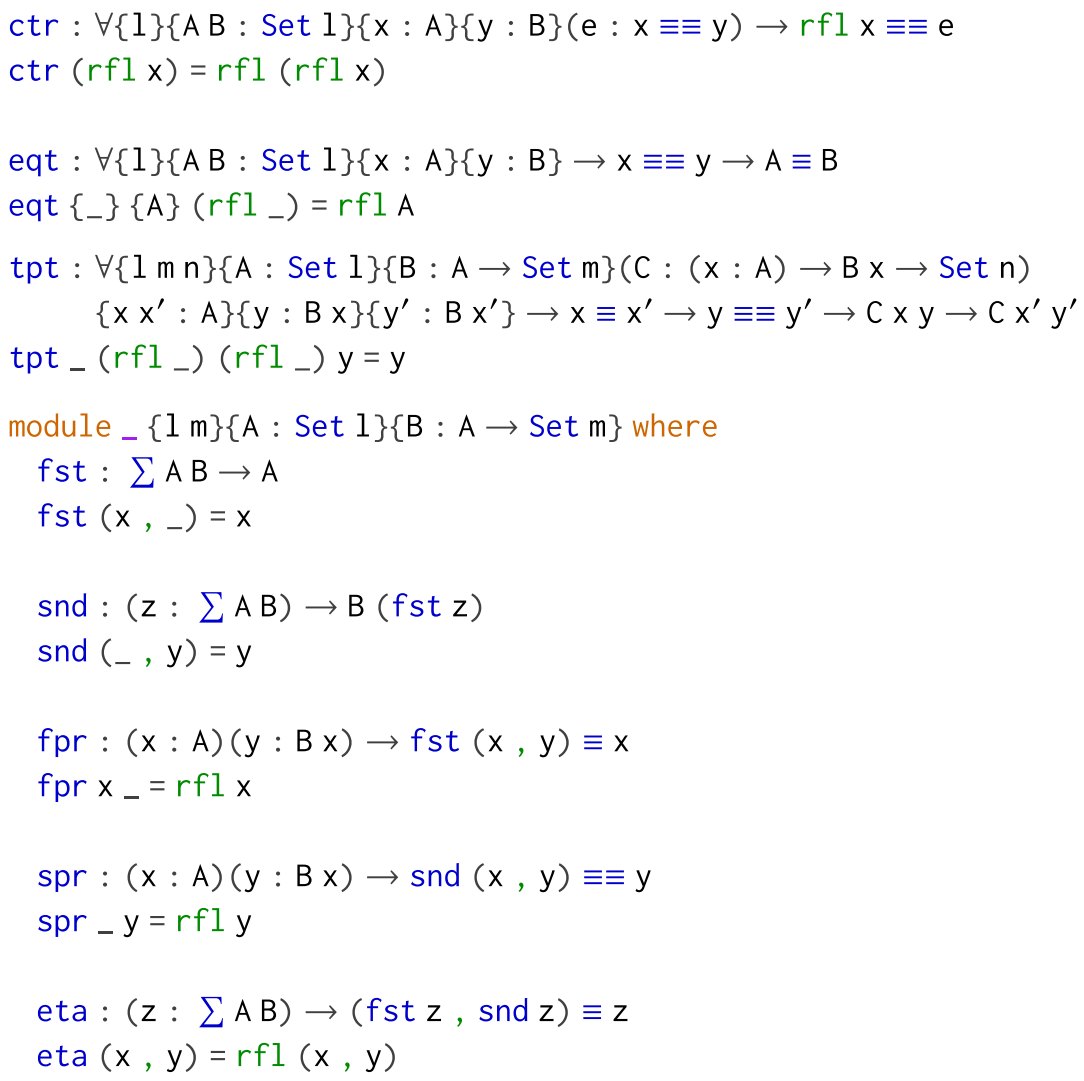

Since we know from the previous section that these functions entail Axiom K, the above definitions have to use Agda's default --with-K option to switch the existing implementation of dependent pattern matching [6] back to the original version due to Reference [8], which is known 
to imply Axiom K [10]. More precisely, it is only the matches on the two occurrences of the pattern $\mathrm{rfl}_{\text {_ }}$ in the definition of tpt that involve an implicit use of Axiom $\mathrm{K}$ (to discharge the unification constraints $\mathrm{A} \doteq \mathrm{A}$ and $\mathrm{B} \mathrm{x} \doteq \mathrm{Bx}$ ); all the other functions can be defined without Axiom $\mathrm{K}$.

\section{CONCLUSION}

This article has investigated heterogeneous equality and produced a simple collection of axioms for its typal form, in the spirit of Reference [9]. The point of view is foundational. From a practical perspective, the use of heterogeneous equality has much to recommend it for formalizing mathematics in dependent type theory when assuming uniqueness of identity proofs ${ }^{1}$; but that is another story.

\section{APPENDIX: TYPAL HOMOGENEOUS EQUALITY WITHOUT K}

In this Appendix, for the sake of completeness, we consider axioms in dependent-type theory without Axiom $\mathrm{K}$ for homogeneous equality types

postulate

$$
\equiv_{-}: \forall\{1\}\{\mathrm{A}: \text { Set } 1\} \rightarrow \mathrm{A} \rightarrow \mathrm{A} \rightarrow \text { Set } 1
$$

following Reference [9]. (Since without Axiom K heterogeneous equality is not very useful, we do not bother to consider axiomatizing $\equiv \equiv$ in that setting.) One of the axioms makes use of dependent product types. Although one could axiomatize those types as we did in the main part of the article, it is simpler to use an inductive defintion and corresponding pair patterns:

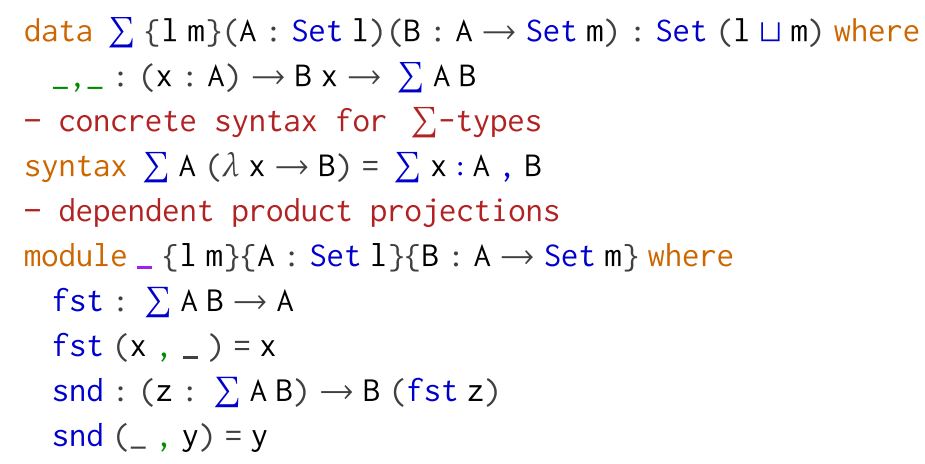

The axioms for homogeneous equality are

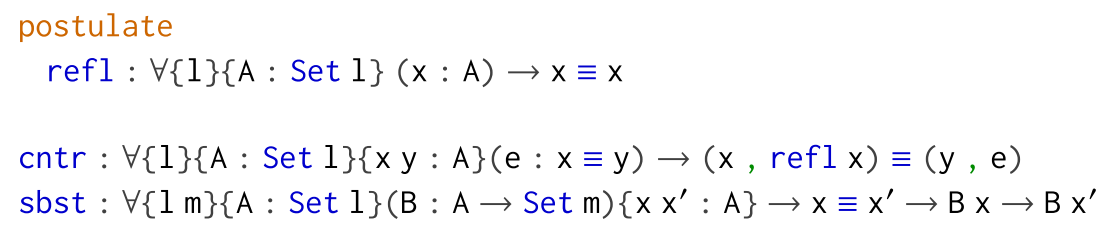

Coquand also considers a regularity axiom for sbst ( $\mathrm{ax}_{3}$ in loc.cit.), but one can do without that by using Peter Lumsdaine's trick to correct sbst to a version subst for which there is a proof

${ }^{1}$ Such is the approach of Lean [16] since version 3, for example. 
substIsRegular $: \forall b \rightarrow$ subst $(r e f l x) b \equiv b$, as follows. The proof begins as for Lemma 2.1 by considering functions that are injective modulo $\equiv$ :

Inj: $\forall\{1\}(A B:$ Set 1$) \rightarrow$ Set 1

$\operatorname{Inj} A B=\sum f:(A \rightarrow B), \forall\{x y\} \rightarrow f x \equiv f y \rightarrow x \equiv y$

id : $\forall\{1\}\{A:$ Set 1$\} \rightarrow A \rightarrow A$

id $x=x$

$\operatorname{idInj}: \forall\{1\}(A:$ Set 1$) \rightarrow \operatorname{Inj} A A$

$i d \operatorname{Inj} j_{-}=(i d, i d)$

But then to construct subst and substIsRegular, one has to work a bit harder than in the proof of the lemma because of the lack of uniqueness of identity proofs:

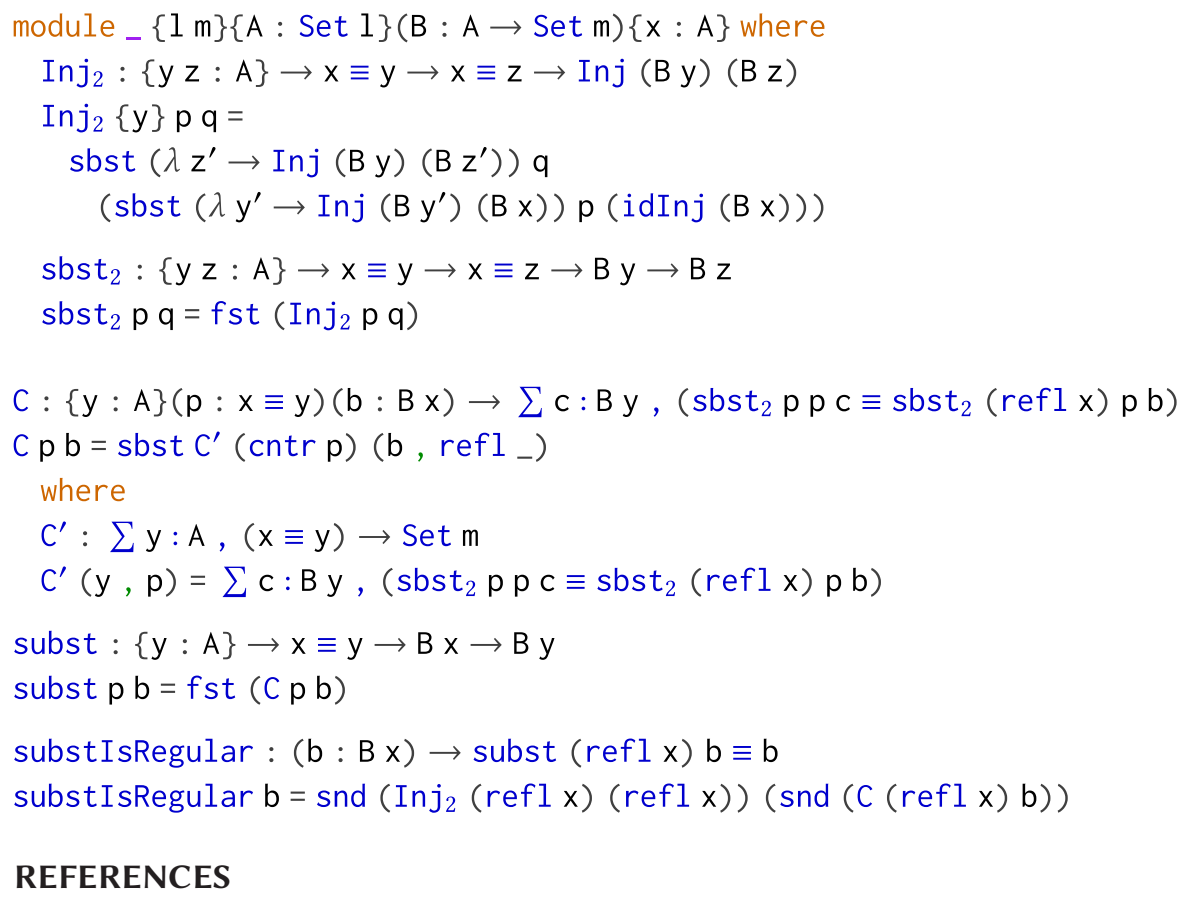

\section{REFERENCES}

[1] Agda Wiki. [n.d.]. Retrieved from https://wiki.portal.chalmers.se/agda/pmwiki.php.

[2] T. Altenkirch, C. McBride, and W. Swierstra. 2007. Observational equality, now! In Proceedings of the Workshop on Programming Languages Meets Program Verification (PLPV'07). ACM, New York, NY, 57-68. DOI : https://doi.org/10. 1145/1292597.1292608

[3] R. Backhouse, P. Chisholm, G. Malcolm, and E. Saaman. 1989. Do-it-yourself type theory. Formal Aspects Comput 1 (1989), 19-84.

[4] M. Bezem, T. Coquand, and S. Huber. 2014. A model of type theory in cubical sets. In Proceedings of the 19th International Conference on Types for Proofs and Programs (TYPES'13) (Leibniz International Proceedings in Informatics (LIPIcs)), R. Matthes and A. Schubert (Eds.), Vol. 26. Schloss Dagstuhl-Leibniz-Zentrum fuer Informatik, Dagstuhl, Germany, 107-128. DOI: https://doi.org/10.4230/LIPIcs.TYPES.2013.107

[5] S. Boulier and T. Winterhalter. 2019. Weak Type Theory is Rather Strong. (June 2019). In Proceedings of the 25th International Conference on Types for Proofs and Programs (TYPES'19). 
[6] J. Cockx and A. Abel. 2018. Elaborating dependent (co)pattern matching. Proc. ACM Program. Lang. 2, ICFP (July 2018). DOI : https://doi.org/10.1145/3236770

[7] C. Cohen, T. Coquand, S. Huber, and A. Mörtberg. 2018. Cubical type theory: A constructive interpretation of the univalence axiom. In Proceedings of the 21st International Conference on Types for Proofs and Programs (TYPES'15) (Leibniz International Proceedings in Informatics (LIPIcs)), T. Uustalu (Ed.), Vol. 69. Schloss Dagstuhl-Leibniz-Zentrum fuer Informatik, Dagstuhl, Germany, 5:1-5:34. DOI : https://doi.org/10.4230/LIPIcs.TYPES.2015.5

[8] T. Coquand. 1992. Pattern matching with dependent types. In Proceedings of the Workshop on Types for Proofs and Programs, B. Nordström, K. Petersson, and G. D. Plotkin (Eds.). 66-79.

[9] T. Coquand. 2011. Equality and Dependent Type Theory. (Feb. 2011). A talk given for the 24th AILA meeting, Bologna. Retrieved from http://www.cse.chalmers.se/ coquand/bologna.pdf.

[10] H. Goguen, C. McBride, and J. McKinna. 2006. Eliminating dependent pattern matching. In Algebra, Meaning, and Computation: Essays Dedicated to foseph A. Goguen on the Occasion of His 65th Birthday, K. Futatsugi, J.-P. Jouannaud, and J. Meseguer (Eds.). Springer, Berlin, 521-540. DOI : https://doi.org/10.1007/11780274_27

[11] C. McBride. 1999. Dependently Typed Functional Programs and their Proofs. Ph.D. Dissertation. University of Edinburgh.

[12] B. Nordström, K. Petersson, and J. M. Smith. 1990. Programming in Martin-Löf's Type Theory. Oxford University Press.

[13] Chr. Paulin-Mohring. 1993. Inductive definitions in the system Coq; rules and properties. In Proceedings of the Conference on Typed Lambda Calculus and Applications (Lecture Notes in Computer Science), M. Bezem and J. F. Groote (Eds.), Vol. 664. Springer-Verlag, Berlin, 328-345.

[14] M. Shulman. 2018. Brouwer's fixed-point theorem in real-cohesive homotopy type theory. Math. Struct. Comput. Sci. 28 (2018), 856-941.

[15] T. Streicher. 1993. Investigations into Intensional Type Theory. Habilitation Thesis. Ludwig Maximilian University, Munich.

[16] The Lean Theorem Prover. [n.d.]. Retrieved from https://leanprover.github.io.

[17] The Univalent Foundations Program. 2013. Homotopy Type Theory: Univalent Foundations for Mathematics. Retrieved from http://homotopytypetheory.org/book, Institute for Advanced Study.

[18] B. van den Berg. 2018. Path categories and propositional identity types. ACM Trans. Comput. Logic 19, 2 (June 2018), 15:1-15:32. DOI : https://doi.org/10.1145/3204492

Received July 2019; accepted January 2020 